\title{
Bioinformatics Analysis of the Phytoene Dehydrogenase Gene in Cabbage (Brassica Oleracea Var. Capitata)
}

\author{
Bo Sun, Min Jiang, Shengling Xue, Aihong Zheng, Fen Zhang and \\ Haoru Tang ${ }^{a, *}$ \\ College of Horticulture, Sichuan Agricultural University, Chengdu 611130, China. \\ *, ahtang@sicau.edu.cn
}

\begin{abstract}
Phytoene dehydrogenase (PDS) is an important enzyme in carotenoid biosynthesis. Here, the Brassica oleracea var. capitata PDS (BocPDS) gene sequences were obtained from Brassica database (BRAD), and preformed for bioinformatics analysis. The BocPDS1 and BocPDS2 genes mapped to chromosomes 3 and 4, and contains an open reading frame of 1,698 bp and 1,692bp that encodes a 565 and 563 amino acid protein, respectively. Subcellular localization predicted both BocPDS1 and BocPDS2 were in the chloroplast. The conserved domain of the BocPDS1 and BocPDS2 proteins is PLN02612. Homology analysis indicates that the levels of identity between BocPDS1 and BocPDS2 were 94\%, and the PDS protein is apparently conserved during plant evolution, and BocPDSs are most closely related to Cruciferae plants. The findings of the present study provide a molecular basis for the elucidation of PDS gene function in cabbage.
\end{abstract}

Keywords: Phytoene dehydrogenase; bioinformatics analysis; Subcellular localization.

\section{Introduction}

Cabbage (Brassica oleracea var. capitata) is a member of the Brassicaceae family that is widely distributed in the world. In China, cabbage is an important vegetable crop, and consumed considerable every years. Cabbage is generally grown for its leafy head as common edible part, which are crispy, tender, and tasty [1]. Besides its good flavor, cabbage is also a rich source of nutrients, antioxidants, and anticarcinogenic compounds, including carbohydrates, vitamin C, glucosinolates, and carotenoids [1-2].

Carotenoids, which are synthesized in various photosynthetic and non-photosynthetic organisms, including algae, plants, and some bacteria and fungi, are a class of 40-carbon hydrocarbon compounds derived from a terpenoid precursor [3-5]. Carotenoids represent a diverse group of pigments that contribute to the red, orange, and yellow colors of various horticultural plants, contributing to their economic value [5-6]. Carotenoids participate in various plant physiological processes, including growth, development, and responses to multiple environmental factors. In green tissues, these act as accessory pigments for the assembly of photosystems and light-harvesting antenna complexes and also have photoprotective functions during photosynthesis $[3,5]$. In non-green tissues, these confer distinct colors to specialized plastids known as chromoplasts, which contribute to the sundry colors in plants $[4,6]$. In addition, carotenoids are the precursors of numerous volatile flavor compounds and phytohormones such as abscisic acid (ABA) and strigolactones [4]. Carotenoids are also essential compounds of human diets, primarily as a precursor of vitamin A [7]. Moreover, carotenoid intake could also reduce the risk for cancer and cardiovascular disease [8-9].

PDS is one of the essential enzymes in carotenoid biosynthesis, catalyzing the colorless phytoene into the $\zeta$-carotene [10]. In addition, it is required for the biosynthesis of chlorophyll. Disruption of PDS will cause an albino phenotypes that can be easily recognized. Thus, PDS is usually to be the target report gene in the experiments of Virus-induced gene silencing (VIGS) and CRISPR/Cas9 gene editing [11-12]. The genes encoding the PDS protein have been isolated in various plant species, including tomato [13], citrus [14], strawberry [15], Strelitzia reginae [16], and Chinese kale [12]. To date, research studies on PDS in cabbage are limited. In the present study, the PDS gene sequences of cabbage were obtained from web database, and then bioinformatics analysis of the PDS genes were analyzed. The present study aimed to establish the foundation for further studies on the molecular mechanism of PDS in cabbage. 


\section{Materials and Methods}

\subsection{Sequence Obtain of the BocPDS Gene}

The genomic DNA and mRNA sequences of PDS gene of cabbage were downloaded and obtained from The Brassica database (BRAD) (http://brassicadb.org), and then used to subsequent bioinformatic analysis.

\subsection{Bioinformatics Analysis of the BocPDS Gene}

The amino acid sequence, protein molecular weight, isoelectric point, stability index, and hydrophobicity of the BocPDS gene were analyzed and predicted by ExPASy (http://web.expasy.org) and NCBI (https://www.ncbi.nlm.nih.gov/). Subcellular localization was predicted by WoLF PSORT (http://www.genscript.com/wolf-psort.html). The conserved domain were predicted by NCBI (https://www.ncbi.nlm.nih.gov/Structure/cdd/wrpsb.cgi). The amino acid sequence of PDS from eight additional species were downloaded from NCBI and subjected to multiple sequence alignment using DNAMAN. Phylogenetic tree analysis of the PDS proteins was executed in MEGA 6.0 using the neighbor-joining (NJ) method.

\section{Results}

\subsection{Analysis on Genomic Organization}

The Brassica database (BRAD) was used to analyze the chromosomal localization and genomic organization of BocPDS. There are two genes of PDS in cabbage chromosomes, BocPDS1 and BocPDS2, and the gene IDs in BRAD are Bol016089 and Bol009962, respectively. The BocPDS1 gene was mapped to chromosomes 3 and has 13 exons and 12 introns, while the BocPDS2 gene was mapped to chromosomes 4 and has 14 exons and 13 introns (Fig. 1).
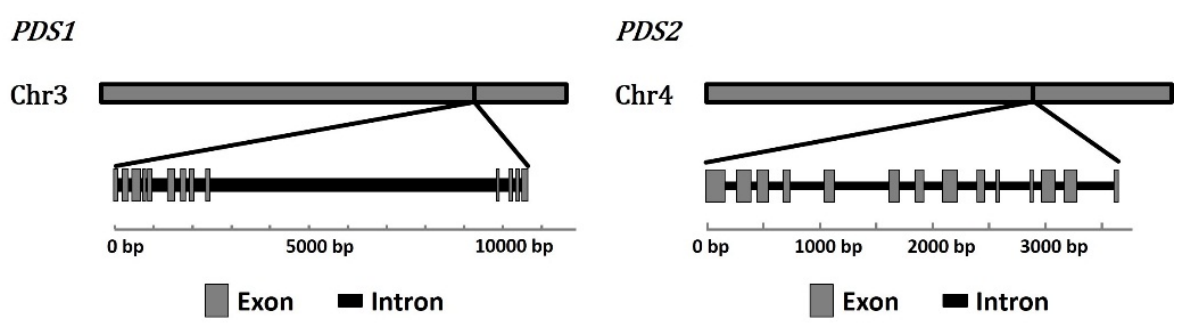

Figure 1. Chromosomal Location and Genomic Structure of BocPDS1 and BocPDS2.

\subsection{Protein Physical and Chemical Properties Analysis}

Sequence analysis indicated that the BocPDS1 and BocPDS2 contained a 1,698-bp and 1,692-bp open reading frame (ORF), which encoded a 565 and 563 amino acids protein with a calculated molecular mass of $63.28 \mathrm{kD}$ and $63.15 \mathrm{kD}$, and an isoelectric point (pI) of 7.11 and 5.96, respectively. The amino acid types and proportions of the BocPDS1 and BocPDS2 was shown in Figure 2, the highest number of amino acid in each gene is Leucine (Leu), whereas the lowest number is Cysteine (Cys). The predicted formula of BocPDS1 and BocPDS2 were C2855H4490N756O819S24 and $\mathrm{C} 2847 \mathrm{H} 4470 \mathrm{~N} 754 \mathrm{O} 822 \mathrm{~S} 23$, respectively. Their total average hydrophilicity index were -0.151 and -0.149 , liposoluble index were 91.35 and 93.04, and instability index in solution were 41.85 and 42.17, respectively. 


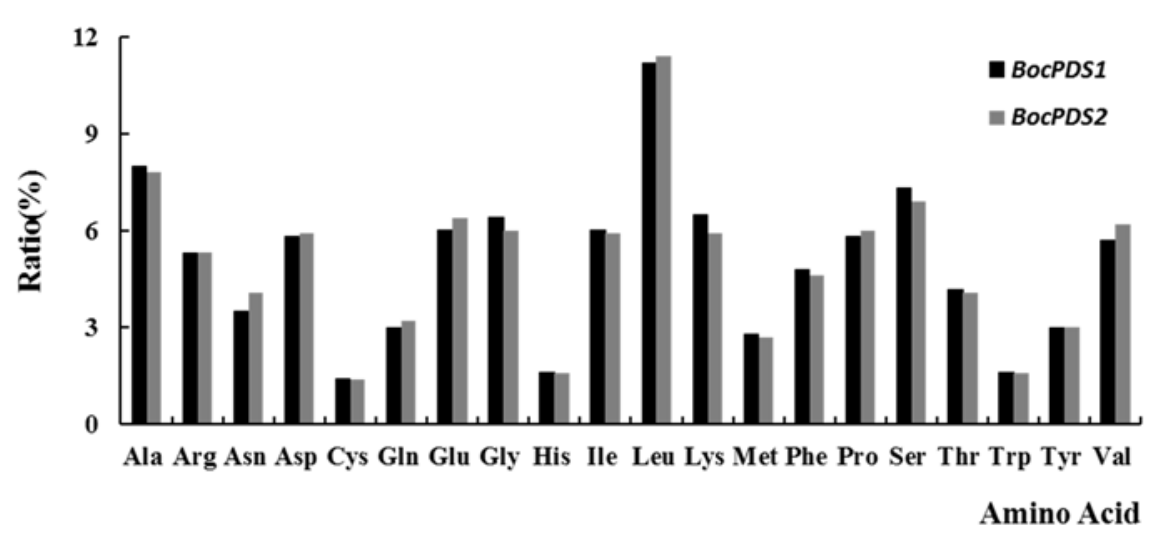

Figure 2. Amino Acid Composition of BocPDS1 and BocPDS2.

\subsection{Subcellular Localization and Conserved Domain Analysis}

Subcellular localization of BocPDS1 and BocPDS2 was both predicted by WoLF PSORT to be in the chloroplast. The analysis using Conserved Domain Database (CDD) demonstrated that the amino acid sequence of both the BocPDS1 and BocPDS2 proteins belong the member of phytoene dehydrogenase, and have one conserved domain PLN02612 and one superfamily COG2907 with predicted NAD/FAD-binding protein structure.

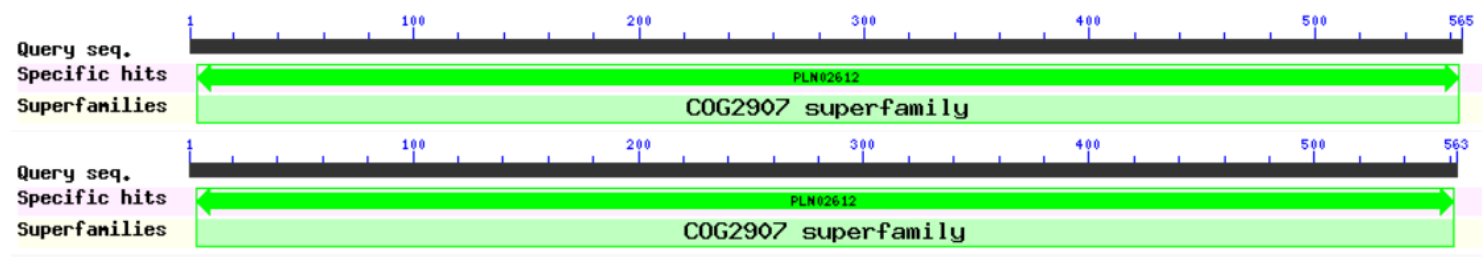

Figure 3. Conserved Domains Analysis of BocPDS1 and BocPDS2.

\subsection{Homology and Phylogenetic Tree Analysis}

Homology analysis demonstrated that the amino acid sequence of the BocPDSs protein shared high homology with those of other higher plant species. The levels of identity between BocPDS1 and BocPDS2 were 94\%. Figure 4 shows that Both BocPDSs had the highest identities with several PDS proteins of Cruciferae such as B. napus, B. rapa, Arabidopsis thaliana, and all of the levels of identity were $>91 \%$. Interestingly, BocPDSs showed $>78 \%$ identity with other species cited in our study, indicating that the PDS protein is highly conserved among different species.

A phylogenetic tree was constructed to illustrate the relationship among the PDS proteins of cabbage and 38 other higher plant species (Fig. 5). Sequence alignment indicated that BocPDS1 protein is more closely related to B. napus, B. oleracea var. alboglabra, B. oleracea var. oleracea, Raphanus sativus, Capsella rubella, and Camelina sativa, while BocPDS2 protein is more closely related to B. oleracea var. botrytis, B. rapa subsp. oleifera, and B. rapa, which all belonged to the Cruciferae branch. 

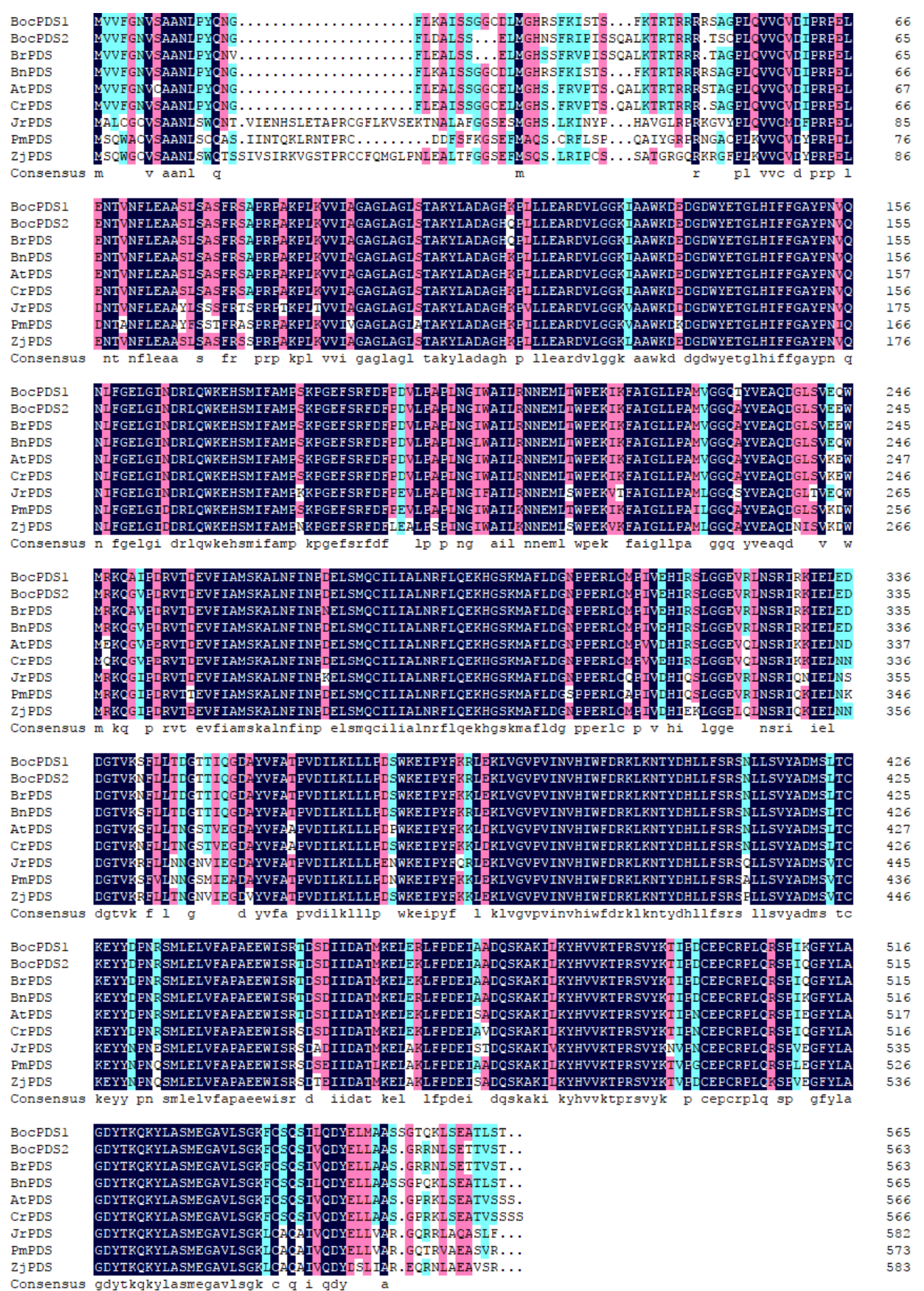

Figure 4. Amino Acid Sequence Alignment of BocPDSs with the PDS Protein of other Species.

Boc: Brassica oleracea var. capitata; Br: Brassica rapa(ACT20709.1); Bn: Brassica napus(NP_001302666.1); At: Arabidopsis thaliana(AAL15300.1); Cr: Capsella rubella(X̄P_006283428.1); Jr: Juglans regia(XP_018828238.1); Pm: Prunus mume(XP_008222708.1); Zj: Ziziphus jujuba(XP_015887115.1). 


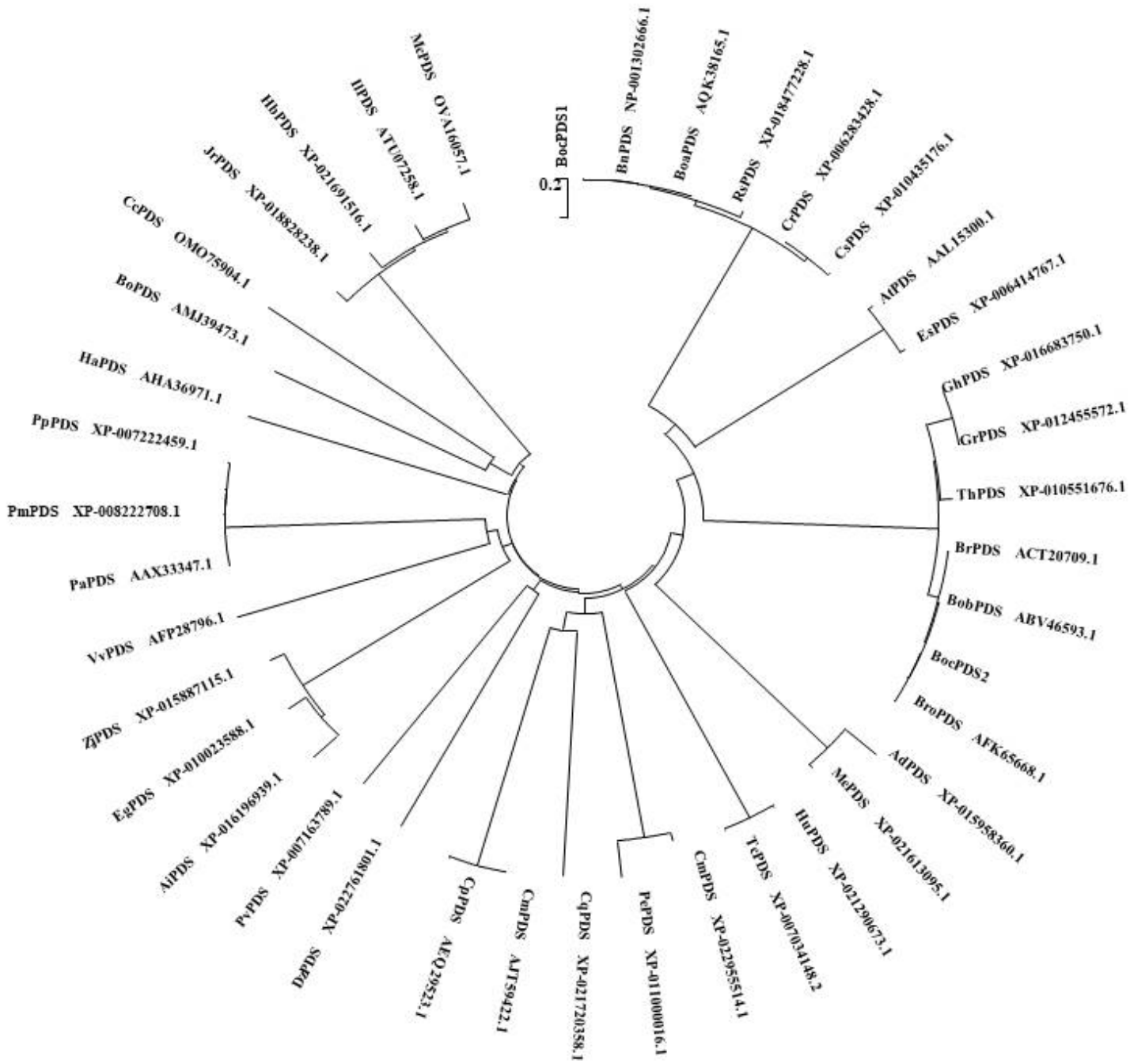

Figure 5. Phylogenetic Tree Analysis of BocPDSs and PDS Proteins of other Species

\section{Discussion}

The present study analyzed the BocPDS genes of cabbage. Both of PDS and ZDS enzymes are encoded by single-copy gene in tomato, grape, and Arabidopsis [5-6]. However, the PDS and ZDS genes respectively occurred as two and a single copy in cabbage, respectively, indicating that the two enzymes may have undergone different evolutionary patterns [12]. Previous studies have shown that the PDS protein is relatively conserved in plants [15-16]. The PDS protein of strawberry is similar to the PDS protein of Prunus armeniaca and Carica papaya, showing $83 \%$ and $83 \%$ homology [15], and PDS of Strelitzia reginae exhibits $86 \%$ homology that of Citrus $\times$ paradise [16]. The findings of the present study show that PDS from cabbage is highly conserved, particularly in plants; in the Cruciferae, these proteins show $>93 \%$ homology, similar to that observed in earlier reports. The findings of the present study may serve as a foundation for future studies on the functions of PDS in carotenoid metabolism in cabbage.

\section{Acknowledgments}

This work was supported by National Natural Science Foundation of China (31500247), key project of Department of Education of Sichuan Province (14ZA0016), and National Student Innovation Training Program (201710626030). 


\section{References}

[1]. M. Wennberg, J. Ekvall, K. Olsson, and M. Nyman, Changes in carbohydrate and glucosinolate composition in white cabbage (Brassica oleracea var. capitata) during blanching and treatment with acetic acid, Food Chem. 95 (2006) 226-236.

[2]. S. Rokayya, C.J. Li, Y. Zhao, Y. Li, and C.H. Sun, Cabbage (Brassica oleracea L. var. capitata) phytochemicals with antioxidant and anti-inflammatory potential, Asian Pac. J. Cancer Prev. 14 (2014) 6657-6662.

[3]. L. Pizarro, and C. Stange, Light-dependent regulation of carotenoid biosynthesis in plants, Cien. Inv. Agr. 36 (2009) 143-162.

[4]. L.H. Liu, Z.Y. Shao, M. Zhang, and Q.M. Wang, Regulation of carotenoid metabolism in tomato, Mol. Plant 8 (2015) 28-39.

[5]. N. Nisar, L. Li, S. Lu, N.C. Khin, and B.J. Pogson, Carotenoid metabolism in plants, Mol. Plant 8 (2015) 68-82.

[6]. P.A. Tuan, J.K. Kim, J. Lee, W.T. Park, D.Y. Kwon, Y.B. Kim, H.H. Kim, H.R. Kim, and S.U. Park, Analysis of carotenoid accumulation and expression of carotenoid biosynthesis genes in different organs of Chinese cabbage (Brassica rapa subsp. pekinensis), Excli J. 11 (2012) 508516.

[7]. N.I. Krinsky, and E.J. Johnson, Carotenoid actions and their relation to health and disease, Mol. Aspects. Med. 26 (2005) 459-516.

[8]. S.T. Mayne, Beta-carotene, carotenoids, and disease prevention in humans, FASEB J. 10 (1996) 690-701.

[9]. F. Giovannucci, Tomatoes, tomato-based products, lycopene, and cancer: review of the epidemiologic literature, J. Natl. Cancer Inst. 91 (1999) 317-331.

[10]. S. Tong, H.X. Xi, K.J. Ai, H. Hou, Cloning and expression analysis of UpPDS gene in Ulva pertusa, Genes Genom. 38 (2015) 285-292.

[11]. B. Zhang, X. Yang, C.P. Yang, M.Y. Li, Y.L. Guo, Exploiting the CRISPR/Cas9 system for targeted genome mutagenesis in Petunia, Sci. Rep. 6 (2015) 20315.

[12]. B. Sun, F. Zhang, X. Xia, S.L. Xue, Q. Yuan, Q. Chen, H.R. Tang, Cloning and expression analysis of BaPDS1 and BaPDS2 in Brassica alboglabra, Acta Horticulturae Sinica 43 (2016) $2257-2265$.

[13]. V. Mann, I. Pecker, J. Hirschberg, Cloning and characterization of phytoene desaturase (Pds) gene from tomato (Lycopersicon esculentum), Plant Mol. Biol. 24 (1994) 429-434.

[14]. M. Kita, A. Komatsu, M. Omura, M. Yano, Y. Ikoma, T. Moriguchi, Cloning and expression analysis of CitPDS1, a gene encoding phytoene desaturase in citrus, Biosci. Biotech. Bioch. 65 (2001) 1424-1428.

[15]. H.S. Zhu, Y.P. Li, Q.F. Wen, and H. Liu, Cloning and characterization of pds gene in Fragaria $\times$ ananassa, Acta Horticulturae Sinica 38 (2013) 55-60.

[16]. M.L. Huang, and R.H. Fan, Cloning and expression analysis of phytoene desaturase in Strelitzia reginae Banks, Acta Horticulturae Sinica 40 (2013) 373-379. 